\title{
BMJ Open Transitions in Parkinson's disease in primary care: protocol of a longitudinal mixed methods study
}

\author{
Annette O A Plouvier, ${ }^{1}$ Tim C Olde Hartman, ${ }^{1}$ Chris van Weel, ${ }^{1,2}$
} Bastiaan R Bloem, ${ }^{3}$ Antoine L M Lagro-Janssen ${ }^{1}$

To cite: Plouvier AOA, Olde Hartman TC, van Weel C, et al. Transitions in Parkinson's disease in primary care: protocol of a longitudinal mixed methods study. BMJ Open 2015;5: e007171. doi:10.1136/ bmjopen-2014-007171

- Prepublication history for this paper is available online. To view these files please visit the journal online (http://dx.doi.org/10.1136/ bmjopen-2014-007171)

Received 11 November 2014 Revised 17 April 2015 Accepted 20 April 2015

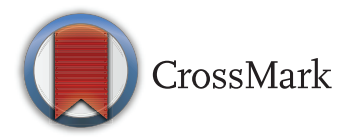

${ }^{1}$ Department of Primary and Community Care, Radboud university medical center, Nijmegen, The Netherlands ${ }^{2}$ Australian Primary Health Care Research Institute, Australian National University, Canberra, Australia ${ }^{3}$ Department of Neurology, Parkinson Center Nijmegen, Donders Institute for Brain, Cognition and Behaviour, Radboud university medical center, Nijmegen,

The Netherlands

\section{Correspondence to} Annette 0 A Plouvier; Annette.Plouvier@ radboudumc.nl

\section{ABSTRACT}

Introduction: Parkinson's disease affects many aspects of the lives of patients and their relatives. Patients must adapt continuously to disabilities that necessitate changes in (medical) support, such as domestic adjustments, involvement of (non) professional caregivers or admission to hospital. Such changes mark a transition: a transfer of a patient between levels or locations of care. Transitions are likely to be multifold and complex, given that Parkinson's disease care extends across all echelons of healthcare. Patients and relatives are vulnerable during a transition, which imposes risks for their safety and quality of life. Guidance by the general practitioner, who knows the preferences of the patient, can help to overcome challenges associated with a transition. However, patient-centred primary care requires insight into the transitions patients with Parkinson's disease encounter. We aim to examine these transitions and the way patients, relatives and general practitioners experience them and cope with them. Moreover, we will study the patients' expectations of their general practitioner during a transition and the general practitioners' views on their role.

Methods and analysis: A longitudinal mixed methods study will be conducted, using qualitative research methods combined with quantitative data as a validated questionnaire on quality of life. Patients will be asked to make a video diary every 2 weeks for a period of 1 year. Once they encounter a transition, patients and their general practitioners will be interviewed to identify causes and consequences of the transition. The verbatim transcripts of the videos and interviews will be analysed according to the principles of constant comparative analysis.

Ethics and dissemination: Ethical approval was not needed according to Dutch legislation. Informed consent of patients, relatives and general practitioners will be obtained. We will disseminate the results in peer-reviewed journals, at research conferences and on the website of the Dutch Parkinson's Disease Association.

\section{INTRODUCTION}

Parkinson's disease (PD) is a neurodegenerative, disabling disease characterised by motor

\section{Strengths and limitations of this study}

- This study will provide insight into crucial elements of transitions in the course of Parkinson's disease, thereby enabling improvement of patient-centred primary care.

- The longitudinal design of the study enables interviewing at turning points in life rather than at fixed moments. Moreover, it allows for withinpatient comparison of experiences and preferences expressed over time.

- The results of the study will need to be interpreted in the light of the studied sample of patients, relatives and general practitioners, and the context and culture of the Dutch healthcare system.

symptoms and a wide variety of non-motor symptoms. ${ }^{1}$ It affects physical, emotional and psychosocial aspects of the lives of patients and their relatives. ${ }^{2}{ }^{3}$ The clinical presentation and rate of progression of PD vary considerably among patients, ${ }^{4}{ }^{5}$ as does the perception of the most troublesome problems. ${ }^{6} 7$ The complexity of the disease requires a multidisciplinary approach with active participation of the patient. ${ }^{8}$ However, due to progression of PD and daily fluctuations of the symptoms, patients and relatives are forced to adapt continuously to new disabilities and limitations in daily life. ${ }^{39}$ Some of these disabilities and limitations necessitate changes in the support or medical care that is offered to a patient. Such changes mark a transition: a transfer of a patient between different levels of (non)professional care within the same location or between different locations of care. ${ }^{10}$ Commonly encountered transitions are the need for domestic adjustments or specific tools for the patient, modification of pharmacotherapy, alternation in the involvement of (non)professional caregivers, adaptation of working hours or type of work and/or admission to specialised day care or hospital. Transitions are likely to be multifold and complex, given that PD care typically extends across all 
echelons of healthcare. ${ }^{911}$ These pose challenges to the communication skills and coping competencies of patients and professionals, since transitions emphasise the need for clarity in the preferences, expectations and roles of everyone involved. Moreover, transfers between different locations of care jeopardise continuity of care. ${ }^{12}{ }^{13}$ Patients and relatives are particularly vulnerable and might feel overwhelmed by a transition, that is often unforeseen. ${ }^{14}$ Safety and quality of life of patients and relatives are at risk. ${ }^{12} 13$

The challenges associated with a transition can be partly overcome by a healthcare professional who is well aware of the care preferences of patients and relatives and who could guide them during a transition, if patients and relatives feel this need. ${ }^{12}{ }^{15}$ In the Netherlands, the general practitioner (GP) is the preferred healthcare professional to fulfil this role as all patients are registered with a general practice; the GP coordinates access to specialised care. ${ }^{16}$ This structure supports Dutch GPs to function as a family doctor, with insight into the physical and mental state of all family members and the contextual factors that influence their well-being. Furthermore, the GP has a long-term professional relationship with the patients and their relatives. No more than $20 \%$ of all patients with PD in the Netherlands are admitted to a nursing home somewhere in the course of their disease. ${ }^{17}$ Over time, the vast majority of patients will, therefore, consult their GP with all sorts of health questions, thereby providing several occasions to discuss the expectations and preferences of the patient and the relatives. ${ }^{18}$ Earlier research in the Netherlands showed that patients with a chronic disease (such as PD) appreciate a long-term relationship with their GP and his/her coordinating role. ${ }^{19}$ Moreover, GPs have been shown to be aware of the need for customised, preferably proactive, care for chronically ill patients. ${ }^{15}$

Such proactive patient-centred primary care requires more insight into the transitions that patients with PD and their relatives encounter during the course of the disease. Therefore, this study aims to answer the following questions:

1. What transitions do patients with PD encounter? How do patients, relatives and GPs experience and cope with these transitions and what is the impact on patients' lives?

2. What are the signs and symptoms of an upcoming transition?

3. What do patients with PD expect from their GP during a transition? Do GPs agree with these expectations?

\section{METHODS AND ANALYSIS}

\section{Study design}

A longitudinal mixed methods study ${ }^{20}$ will be performed, using qualitative research methods as video diaries and in-depth interviews and quantitative data as a validated questionnaire on quality of life and ratings for the neurological signs of PD.

\section{Participants}

\section{General practitioners}

A purposive sample of general practices surrounding Nijmegen, the Netherlands, will be approached to participate. Purposive sampling will be used to increase the external validity and to provide a wide range of opinions. Based on expert experience and literature, we consider the following characteristics to be relevant for purposive sampling: geographical location of the practice (city vs rural area); practice organisation (group practice vs solo practice); age, gender and working experience of the GP. GPs will be approached to participate until saturation in data analysis is reached.

\section{Patients}

The patient population will consist of patients with PD. In order to participate, patients need to meet the following inclusion criteria:

- The diagnosis of PD is confirmed by a neurologist, according to established guidelines. ${ }^{21}$

- The patient lives independently, possibly with help from (non) professional caregivers.

- The patient does not have a form of cognitive dysfunction (according to the GP) and is therefore, mentally capable of remembering what happened in the past weeks/months.

- The patient is capable of handling a simple video camera with clear instructions (possibly with help of a partner or significant other).

For each participating patient, the partner or a significant other will be asked to participate as well.

Inclusion of patients (preferably with diversity in gender, age, and Hoehn-Yahr stage of PD), data collection and data analysis will continue iteratively until saturation is reached.

\section{Recruitment}

\section{General practitioners}

GPs will be personally approached to participate. Subsequently, an email will be sent in which the aim of the study is explained briefly and more information is given to inform the GP what participation means for the GP, for the patient and for the partner/significant other.

Once a GP confirms to participate, he/she will search the computerised patient files of the general practice to identify patients with PD that fit the inclusion criteria.

\section{Patients}

Patients that fit the inclusion criteria described above will initially be approached by their GP. The GP will give a brief explanation of the study and will ask if the patient is willing to be approached by the researcher. If so, the GP will inform the researcher and the researcher will contact the patient in order to send an information letter. A week later, the researcher will approach the patient again and will ask for questions regarding the study. When a patient (and possibly the partner/significant other) agrees to participate, an appointment will 
be made with the researcher in order to give more detailed information on the study and to explain the use of the camera. Moreover, this appointment will serve to ask the patient (and possibly the partner/significant other) for informed consent. Patients consenting participation can be included even when their partner/significant other does not consent to participate.

\section{Data collection}

Video diaries

Patients will be asked to make a video message of 5-10 min every 2 weeks for a period of 1 year. A pilot study already proved the feasibility of this method. Patients will use a basic video camera (JVC Pics 10, Sony MHS-FS1 or Panasonic HC-V110) that is provided to them for the purpose of this study. Patients will make the videos themselves in their own home, supported by an instruction manual. This manual includes an instruction on the use of the video camera and instructions regarding the content of every video message. A video message has to contain the following items:

- The name of the patient and the date of the recording.

- A grade between 0 and 10 ( 0 being the worst imaginable, 10 the best), reflecting the way the patient felt in the 2 weeks before the recording.

- Two tests for neurological signs of PD:

- Finger tapping test, executed to conform to ' 3.4 Finger tapping' of the Movement Disorder Societysponsored revision of the Unified Parkinson's Disease Rating Scale (MDS-UPDRS): ${ }^{22} 40$ times with the left hand and 40 times with the right hand.

- Arising from the chair, executed to conform to ' 3.9 Arising from chair' of the MDS-UPDRS. ${ }^{22}$

- A description of three situations, that happened in the 2 weeks before the recording:

- A situation that went very well, despite the fact that the patient has PD. What was the reason this went well?

- A situation that did not work out the way the patient expected it to because of PD. What was the reason for it?

- A situation in which the patient got help because of PD. Why was help necessary? Was the help asked for or offered? How did the patient experience the need for help and how did the patient experience the help itself?

When the patient's partner/significant other has agreed to participate in the study as well, he/she will be asked for a subsequent video message (following the recording of the patient) containing:

A description of a situation, that happened in the 2 weeks before the recording, in which the partner/significant other gave help to the patient with PD. Did he/she offer to help or did the patient ask for it? How did it feel to help? And in general, how does it feel to be a caregiver?
Once a month, a research assistant will collect the video diaries. The assistant and the patient will also fill in the PDQ39, (a validated Dutch version of) a questionnaire on the quality of life of patients with PD. ${ }^{23-25}$ Furthermore, the research assistant will explore whether a transition has taken place in the last month, using a short questionnaire in which the patient will be asked if any of the transitions, which are the focus in this study, have taken place.

\section{Identification of a transition}

A transition is defined as the transfer of a patient between different levels of (non) professional care within the same location or between different locations of care. ${ }^{10}$ For this study, we will focus on a number of specific transfers, given they occur as a consequence of PD:

- A change in the extent of domestic help that is provided.

- A change in the extent of help that is necessary for personal care.

- A domestic adjustment (such as a bracket on the toilet or shower).

- The purchase of a specific tool (such as a walker or adapted cutlery).

- A modification of pharmacotherapy.

- The involvement of a healthcare provider (including, eg, the physical therapist and speech therapist), who was not involved before.

- Consultation of the GP or medical specialist, if not part of a routine follow-up.

- Adaptation of working hours or type of work.

- Admission to specialised day care or hospital.

\section{In-depth interviews}

Once a patient encounters a transition, he/she will be contacted to participate in an in-depth interview. The interviewer will stimulate the patient to tell more about the transition and the way the patient handled it. A brief topic list will be made to guide the interviewer, possible topics are the patient's view on forerunner signs and causes of the transition, the role of caregivers during the transition and the impact of the transition on the life of the patient and his/her partner. As data collection and analysis will proceed as an iterative process, relevant and new topics will be added to the topic list after a preliminary analysis of every interview. In this way, ideas and thoughts that emerge in primary stages of the analysis will be brought forward in subsequent interviews as the study proceeds, in order to reach a deeper understanding of the relevant topics and themes.

The patient's GP will be approached for an in-depth interview on the transition as well. A different topic list will be used for the interviews with GPs, focusing on forerunner signs and causes of the transition, the role of the GP and the patient's prognosis concerning transitions due to PD. This topic list will also be modified according to the iterative process described above. GPs 
will be asked verbally (recorded on tape) for informed consent prior to the interview.

All interviews will be recorded on tape for the purpose of transcription.

\section{Data analysis}

The researcher will watch all recorded videos. Once a transition has taken place, the researcher will review the last four videos of the patient involved for cues of an upcoming transition and for specific details to be discussed in the interviews. The reviewed videos will be transcribed verbatim for analysis, ensuring the anonymity of the patient. Speech, facial expression, finger tapping and arising from the chair will be scored according to the MDS-UPDRS points 3.1, 3.2, 3.4 and 3.9, respectively. ${ }^{22}$ PDQ-39 will be scored using the sum score of all subscores. ${ }^{25}$ These sum scores will be plotted in a graph, that expresses the sum score per patient per month and the moment a transition has taken place.

All recorded in-depth interviews will be transcribed verbatim anonymously for analysis as well.

\section{Analysis}

The transcripts of the video diaries and in-depth interviews will be entered into ATLAS.ti 7, a software programme for detailed coding in qualitative data analysis. In order to refine and focus the interview topic guides, analysis of the videos and in-depth interviews will start the moment the first transition has taken place and the videos and interviews have been transcribed. The analysis will be according to the principles of constant comparative analysis. ${ }^{26}$ Two researchers will read all transcripts several times to familiarise themselves with the data. They will independently apply codes to meaningful words and sentences in the transcripts. These codes will be discussed, seeking agreement for their content. In case of disagreement, the opinion of a third researcher will be sought. Codes will then be grouped into themes that represent important and relevant aspects of transitions, as formulated in the research questions. Themes will be used to refine the interview topic guides and to progressively focus and explore the data in depth. Analysis will continue until saturation is reached.

\section{ETHICS AND DISSEIMINATION}

Longitudinal research with chronically ill patients poses several ethical issues. ${ }^{27}$ One of these concerns the consent to participate. Patients will be asked for their written consent before they are included in the study. However, unforeseen circumstances and progression of the disease might change a patient's opinion. Consent will therefore be verified verbally before each interview. Moreover, when the researcher or research assistant suspects diminishing enthusiasm to participate, this will be brought up and the patient will be reminded that participation is voluntary and can be ended (preliminary) at any time.
Furthermore, continuity in staff is a key element of longitudinal research. Therefore, we aim to assign one researcher, who will be responsible for watching all videos and performing the in-depth interviews. Moreover, this researcher will be part of the analysing team. We also aim to make sure that the same research assistant will visit the participating patients every month.

The results of this study will be disseminated in peerreviewed journals and at research conferences. Results will also be published on the website and in the magazine of the Dutch Parkinson's Disease Association. Moreover, the patients, relatives and GPs participating in the study will be informed about the results.

\section{DISCUSSION}

In this study we will focus on transitions in PD in patients living at home. We will explore the transitions that patients encounter in the course of their disease and examine the way patients, their partners/significant others and their GPs experience these transitions. Moreover, our results will facilitate the anticipation of upcoming transitions. Finally, this study will offer the opportunity to compare the patients' expectations from their GP during a transition and the GPs' views on their role in a transition. Therefore, this study will provide insight into crucial elements of transitions in the course of $\mathrm{PD}$, such as the extent to which patients want their GP to be involved during a transition and how this could influence their experiences. Moreover, this could give insight into the most ideal moment to offer primary care during a transition. This will enable improvement of (proactive) primary care for patients with $\mathrm{PD}$, in a patient-centred way.

\section{RECRUITMENT CHALLENGES}

Recruiting patients with a chronic disease to participate in longitudinal qualitative research poses difficulties. ${ }^{27}$ Furthermore, recruitment of GPs and patients in primary care can be challenging. ${ }^{28}$ In this study we expect that the challenges at the GP's level include willingness to participate and to fulfil all requests associated with participation: searching the computerised patient files for patients that fit the inclusion criteria, approaching suitable patients, informing the researcher when a patient is willing to be approached, and participating in an in-depth interview.

Moreover, there are possible challenges at the level of the patient. The number of patients with PD in each general practice is about four ${ }^{29}$ and only a selection of these patients will fit the inclusion criteria. One could also argue that the patients that do fit the inclusion criteria are relatively independent patients with PD. It is very well possible that this group of patients will encounter different transitions than patients with more advanced PD. However, the GP is the main caregiver for patients with PD who still live at home; therefore, we specifically aim to study this relatively independent group. If the 
present approach is successful, future work should address the transitions in more advanced PD. Apart from this, patients might find it difficult to agree with participation for a period of a year, as they are uncertain what the next year will bring to them. Unforeseen circumstances could force the patient to stop his/her participation preliminary. Furthermore, participating patients might have difficulties in handling the video camera or describing useful situations in their video recordings. However, a pilot study suggested that patients with PD are able to make useful video recordings themselves by following the instruction manual provided to them.

Challenges at the level of the relative could also influence recruitment, such as the inability to handle an additional, non-essential, task besides care-giving or their opinion that participation in a study might not be good for the patient.

\section{STRENGTHS AND LIMITATIONS}

The home setting, in which the videos will be recorded, might comfort patients and partners to freely express symptoms and difficulties they experience in daily life, even those they might interpret as too unimportant to consult the GP for although these might have a significant impact on the quality of life.

The longitudinal qualitative design of this study provides other unique opportunities. ${ }^{27}$ For example, it will be possible to interview patients and their GPs at turning points in life rather than at fixed moments. Furthermore, the information from the video diaries combined with the iterative process of data analysis enables refinement and customisation of the topic guide for each interview. This will give a deeper understanding of the transitions encountered in the course of PD, and the causes and consequences of these over time. In addition, the results of the in-depth interviews with the patients and their GPs enables comparison between the patient's views and expectations and those of the GP, providing insight into possible discrepancies. Moreover, the video diaries allow for within-patient comparison of the results, since progression of the disease and the constant adaptation to new disabilities or limitations might influence the experiences and preferences patients express over time. Finally, the use of multiple qualitative research methods, supported by quantitative data, offers valuable opportunities for data triangulation. ${ }^{20}$ The constant comparative content analysis, applied until saturation is reached, adds further to the robustness and validity of the results. ${ }^{26}$

However, qualitative research methods have disadvantages as well. Qualitative research has neither the goal nor the suited method to quantify variables or to generalise results from a small sample to a larger population. Therefore, the results of this study will need to be interpreted in the light of the studied sample of patients and GPs, and the context and culture of the Dutch healthcare system.
Contributors CVW, BRB and ALML-J were responsible for the design of the study. AOAP and TCOH participated in the design of the study. AOAP wrote the first draft of the manuscript. TCOH, CVW, BRB and ALML-J revised the manuscript critically. All the authors read and approved the final manuscript.

Funding This study is supported by funds of the Dutch Parkinson's Disease Association (Funding number 2012-V15) and the Health Insurers Innovation Foundation (Funding number 2687).

\section{Competing interests None declared}

Provenance and peer review Not commissioned; externally peer reviewed.

Data sharing statement No additional data are available.

Open Access This is an Open Access article distributed in accordance with the Creative Commons Attribution Non Commercial (CC BY-NC 4.0) license, which permits others to distribute, remix, adapt, build upon this work noncommercially, and license their derivative works on different terms, provided the original work is properly cited and the use is non-commercial. See: http:// creativecommons.org/licenses/by-nc/4.0/

\section{REFERENCES}

1. Goldman JG, Postuma R. Premotor and nonmotor features of Parkinson's disease. Curr Opin Neurol 2014;27:434-41.

2. Schrag A, Jahanshahi M, Quinn N. How does Parkinson's disease affect quality of life? A comparison with quality of life in the genera population. Mov Disord 2000;15:1112-18.

3. Martinez-Martin P, Rodriguez-Blazquez C, Forjaz MJ. Quality of life and burden in caregivers for patients with Parkinson's disease: concepts, assessment and related factors. Expert Rev Pharmacoecon Outcomes Res 2012;12:221-30.

4. Mehanna R, Moore S, Hou JG, et al Comparing clinical features of young onset, middle onset and late onset Parkinson's disease. Parkinsonism Relat Disord 2014;20:530-4.

5. Haaxma CA, Bloem BR, Borm GF, et al Gender differences in Parkinson's disease. J Neurol Neurosurg Psychiatry 2007;78:819-24.

6. Politis M, Wu K, Molloy S, et al Parkinson's disease symptoms: the patient" perspective. Mov Disord 2010;25:1646-51.

7. Louter M, van der Marck MA, Pevernagie DA, et al Sleep matters in Parkinson's disease: use of a priority list to assess the presence of sleep disturbances. Eur J Neurol 2013;20:259-65.

8. Post B, van der Eijk M, Munneke M, et al. Multidisciplinary care for Parkinson's disease: not if, but how! Pract Neurol 2011;11:58-61.

9. van der Marck MA, Bloem BR. How to organize multispecialty care for patients with Parkinson's disease. Parkinsonism Relat Disord 2014;20(Suppl 1):S167-73.

10. Coleman EA, Boult C, American Geriatrics Society Health Care Systems Committee. Improving the quality of transitional care for persons with complex care needs. J Am Geriatr Soc 2003;51:556-7.

11. Temlett JA, Thompson PD. Reasons for admission to hospital for Parkinson's disease. Intern Med J 2006;36:524-6.

12. Coleman EA. Falling through the cracks: challenges and opportunities for improving transitional care for persons with continuous complex care needs. J Am Geriatr Soc 2003;51:549-55.

13. Kripalani S, LeFevre F, Phillips CO, et al. Deficits in communication and information transfer between hospital-based and primary care physicians: implications for patient safety and continuity of care. JAMA 2007;297:831-41.

14. Coleman EA, Smith JD, Frank JC, et al Development and testing of a measure designed to assess the quality of care transitions. Int $J$ Integr Care 2002;2:e02.

15. Hudon C, Fortin M, Haggerty J, et al Patient-centered care in chronic disease management: a thematic analysis of the literature in family medicine. Patient Educ Couns 2012;88:170-6.

16. van Weel $\mathrm{C}$, Schers $\mathrm{H}$, Timmermans $\mathrm{A}$. Health care in the Netherlands. J Am Board Fam Med 2012;25(Suppl 1):S12-17.

17. Riem D, Zuidema S, Bor H, et al [Parkinson's disease]. Tijdschr Verpleeghuisgeneeskunde 2009;34:127-31.

18. Frosch DL, May SG, Rendle KA, et al Authoritarian physicians and patients' fear of being labeled 'difficult' among key obstacles to shared decision making. Health Aff (Millwood) 2012;31:1030-8.

19. Berendsen AJ, de Jong GM, Meyboom-de Jong B, et al Transition of care: experiences and preferences of patients across the primary/ secondary interface-a qualitative study. BMC Health Serv Res 2009;9:62.

20. Borkan JM. Mixed methods studies: a foundation for primary care research. Ann Fam Med 2004;2:4-6. 
21. Bloem BR. [Multidisciplinary guideline Parkinson's disease]. Van Zuiden Communications, 2010.

22. Goetz CG, Tilley BC, Shaftman SR, et al Movement Disorder Society-sponsored revision of the Unified Parkinson's Disease Rating Scale (MDS-UPDRS): scale presentation and clinimetric testing results. Mov Disord 2008;23:2129-70.

23. Martinez-Martin $\mathrm{P}$, Jeukens-Visser $\mathrm{M}$, Lyons KE, et al Health-related quality-of-life scales in Parkinson's disease: critique and recommendations. Mov Disord 2011;26:2371-80.

24. Marinus J, Visser M, Jenkinson $\mathrm{C}$, et al Evaluation of the Dutch version of the Parkinson's Disease Questionnaire 39. Parkinsonism Relat Disord 2008;14:24-7.

25. Peto V, Jenkinson C, Fitzpatrick R. PDQ-39: a review of the development, validation and application of a Parkinson's disease quality of life questionnaire and its associated measures. J Neurol 1998;245(Suppl 1):S10-14.

26. Elo $S$, Kyngäs $H$. The qualitative content analysis process. J AdV Nurs 2008;62:107-15.

27. Calman L, Brunton L, Molassiotis A. Developing longitudinal qualitative designs: lessons learned and recommendations for health services research. BMC Med Res Methodol 2013;13:14.

28. van der Wouden $\mathrm{JC}$, Blankenstein $\mathrm{AH}$, Huibers $\mathrm{MJ}$, et al Survey among 78 studies showed that Lasagna's law holds in Dutch primary care research. J Clin Epidemiol 2007;60:819-24.

29. van de Lisdonk EH, Van den Bosch W, Lagro-Janssen A, et al [Diseases in general practice]. Maarssen: Elsevier Gezondheidszorg, 2008. 